\title{
Detecting Public Health Impacts Associated with Air Pollution Events in the UK Using Syndromic Surveillance
}

\author{
Yolande Macklin*1,2, Andrew Kibble ${ }^{3}$, Alex Elliot ${ }^{4}$ and Gillian Smith ${ }^{4}$ \\ ${ }^{1}$ School of Health and Population Sciences, University of Birmingham, Birmingham, United Kingdom; ${ }^{2}$ Centre for Radiation \\ Chemical and Environmental Hazards, Public Health England, Birmingham, United Kingdom; ${ }^{3}$ Centre for Radiation Chemical and \\ Environmental Hazards, Public Health England, Cardiff, United Kingdom; ${ }^{4}$ Real-Time Syndromic Surveillance Team, Public Health \\ England, Birmingham, United Kingdom
}

\section{Objective}

This study examined whether the current UK real-time syndromic surveillance systems can detect public health impacts associated with air pollution events such as fires and ambient air pollution episodes.

\section{Introduction}

Air pollution is well documented to cause adverse health effects in the population. Epidemiological/toxicological studies have demonstrated that air pollution is associated with various adverse health outcomes, ranging from mortality to subclinical respiratory symptoms [1]. Classical epidemiological studies of the health effects of air pollution are typically retrospective. In order to assess the effectiveness of any public health messages or interventions in a timely manner there is a need to be able to systematically detect any health effects occurring in 'real-time'.

The UK syndromic surveillance systems are coordinated by Public Health England (PHE) and are used to monitor infectious diseases in real-time [2]. This study is the first in the UK to explore whether syndromic surveillance systems can detect public health impacts associated with air pollution events.

\section{Methods}

Retrospective analysis of the syndromic surveillance systems was undertaken using case studies of UK air pollution events including; ambient air pollution episodes, large scale industrial fires and wildfires. Key syndromes were selected which are typical of clinical presentation following exposure to elevated air pollutants such as; asthma, myocardial infarction, wheeze and eye irritation. Potential confounders such as pollen, humidity and temperature which can cause similar syndromes were also considered in the study. Data was extracted from the syndromic surveillance systems for the time period of the air pollution event and included a lag period for delayed onset of symptoms.

Time series graphs and Shewhart control charts were used to identify whether increases in syndromes typical of exposure to air pollution correlated with air pollution events. Where correlation was noted, the historical 'alarm' records within the syndromic surveillance systems were interrogated to determine if these events had been identified as statistically significant.

\section{Results}

The study found that syndromic surveillance has the potential to detect public health impacts associated with certain types of air pollution events. Out of the 13 events studied one ambient air pollution episode and two large scale industrial fires triggered 'alarms'. Other air pollution events showed a strong correlation with a rise in syndromic indicators, but these did not trigger 'alarms' within the syndromic surveillance systems. In particular air pollution events which are either; prolonged, widespread or very large scale were more likely to be detected.
Daily reporting systems and syndromic indicators associated with exacerbation of pre-existing respiratory conditions were more sensitive in detecting public health impacts associated with air pollution events. However, further work is required to refine the statistical methodology which underpins 'alarms' being triggered in the syndromic surveillance systems to enable air pollution events to be detected prospectively.

\section{Conclusions}

This study indicated there is significant potential for syndromic surveillance systems to be used to monitor public health impacts during air pollution events in the UK. It has also highlighted its potential to be used to provide tailored public health messages to the local population during an air pollution event, and assist in preparing and ensuring adequate healthcare resources are mobilised to minimise the impact on public health.

\section{Keywords}

Air pollution; syndromic surveillance; syndromes; retrospective analysis; public health

\section{References}

[1] WHO (2010) Preventing disease through healthy environments. Exposure to air pollution: A major public health concern: Geneva, Switzerland: World Health Organization.

[2] HPA (2012) Real-Time Syndromic Surveillance. Available from: http://www.hpa.org.uk/Topics/InfectiousDiseases/InfectionsAZ/RealtimeSyndromicSurveillance/ [Accessed 06/05 2013].

\footnotetext{
*Yolande Macklin

E-mail: yolande.macklin@phe.gov.uk
} 